\title{
Health Equity and Lifestyle Medicine in the Medical School Curriculum
}

\author{
Gautam Ramesh ${ }^{1}$ and Kyleigh C. Kirbach, MS, RDN ${ }^{1}$ \\ ${ }^{1}$ School of Medicine, University of California, San Diego, La Jolla, CA 92093
}

August 2, 2021

We, as students in the medical field, have the unique privilege of playing an influential role in transforming the system that we are trainees of. It is our prerogative to observe, internalize, scrutinize, and reform the medical curriculum to reflect what we believe will make the largest impact on our patients, and the patients of future students at our institution.

Over the last twelve months, we have seen numerous efforts at medical schools across the nation to push forth a curriculum more mindful of health disparities and racism in medicine. Student-changemakers at our school have spearheaded a multi-year Health Equity curriculum, inspiring faculty to create coursework centered around educating students on social determinants of health, gender and race disparities in care, health outcomes based on age, incarceration, homelessness, and more.

Our own impetus was borne of the realization that the healthcare system has devolved into a diseasecare system. While the overwhelming majority of chronic disease can be altogether prevented through healthy lifestyle behaviors ${ }^{1}$, few Americans engage in adequate physical activity, follow a healthy diet, maintain a healthy weight, and abstain from smoking ${ }^{2}$. These lifestyle deficiencies arise not from laziness; rather, numerous external factors induce these behaviors, such as proximity of grocers and famers markets, availability of safe recreation spaces, presence of community support, racial inequities, and influence of media and advertising.

To educate our peers and the future medical students at our school, we created a seminar series and a health equity lecture to highlight the countless determinants at play when it comes to a patient's health. By emphasizing the importance of lifestyle behaviors in preventing disease, and underscoring how to address these behaviors in a clinical encounter, we aim to erect a pillar of compassion in our curriculum, training future physicians to consider all potential influences on a patient's health, and to advocate for them through the creation of wellness-centered policy.

A gap in medical training is not just a lapse of understanding; it is an instant of substandard patient care that could be better - diseases that could be prevented, and lives that could be saved. By editing the next iteration of medical education, we, as medical students, hold the power to rewrite the future of the health of our country.

Acknowledgements: The authors thank Dr. Deepa Sannidhi, M.D., and Dr. Weena Joshi, M.D., for their guidance and mentorship in these endeavors. 
Funding/Support: None.

Other disclosures: None.

Ethical approval: Not applicable.

Disclaimers: None.

\section{References}

1.Ford, E. S. et al.. Healthy living is the best revenge: findings from the European Prospective Investigation Into Cancer and Nutrition-Potsdam study.. Arch Intern Med 169, 1355-62 (2009).

2.Reeves, M. J. \& Rafferty, A. P. Healthy lifestyle characteristics among adults in the United States, 2000.. Arch Intern Med 165, 854-7 (2005). 